\title{
The Cavitation Resistance of WC-CoCr Cermet Coating Deposited by HVOF for Hydraulic Application
}

\author{
Qian Huang ${ }^{1} \cdot$ Enwei Qin ${ }^{1} \cdot$ Wenli $\mathrm{Li}^{2} \cdot$ Bo Wang ${ }^{1}$. Chenyang Pan ${ }^{1}$. Shuhui $\mathrm{Wu}^{1}$ \\ ${ }^{1}$ Suzhou Nuclear Power Research Institute, Suzhou 215004, China. \\ ${ }^{2}$ School of Mechanical and Electric Engineering \& Collaborative Innovation Center of Suzhou Nano Science and Technology, \\ Soochow University, Suzhou 215123, China.
}

\section{ABSTRACT}

Thermal spray is a versatile technique for enhancing the cavitation resistance of hydraulic water passage components, especially in on-site repair situation. Based on the application in hydraulic components, a WCCoCr cermet coating was deposited by high velocity oxygen fuel spraying. The microstructure and hardness were characterized, and the cavitation was studied by ultrasonic vibratory system according to ASTM G32 standard. The coating shows superior anti-cavitation behavior in term of mass weight loss compared with the martensite stainless steel. The cavitation erosion mechanism is elaborated with wrinkles and craters observed on the worn surfaces, and correlated with the incubation and accelerating stages.

Special Issue on Asian Thermal Spray Conference (ATSC2020)

(C)Indian Thermal Spray Society, INScienceIN. 2020.All rights reserved

ARTICLE HISTORY

Received 18-12-2020

Revised 29-12-2020

Accepted 30-12-2020

Published 05-01-2021

\section{KEYWORDS}

WC-CoCr coating

Cavitation

Erosion

\section{Introduction}

Two-phase fluid of water-vapor exists in hydraulic water passage components. Due to pressure difference, bubbles will collapse implosively and impact on the surface, resulting in materials loss and fatigue. Consequently, flow instability and excessive vibration is induced, which leads to premature failure in mechanical components[1-3]. This phenomenon was initially defined as cavitation by Knapp [4]. Cavitation erosion has been considered as one of the crucial damage problems in hydraulic water passage components. Extensive numerical or experimental studies on cavitation has been carried out in pumps $[5,6]$, guide vanes [7-8], runner blades [9-10], etc.

From the material perspective, the cavitation erosion resistance can be enhanced by selection of alloys with combination of high hardness and excellent toughness. Therefore, martensite AISI 410 stainless steel or precipitation hardened 17-4 $\mathrm{PH}$ stainless steel are the typical materials for hydraulic components such as blades. To further improve the cavitation performance, surface techniques including plating [11-13], spraying [14-18]or cladding [19-20]have been employed to form functional anti-cavitation thin layer over base materials.

Iwai et al [12-13] found out that chromium plating showed better cavitation behavior than carbon steel. They also pointed out the possible galvanic corrosion at the interface between the plating and base materials. Due to the environmental toxicity of the hexavalent chromium in the bath solution, other alternatives including plating $\mathrm{Ni}-\mathrm{W}$

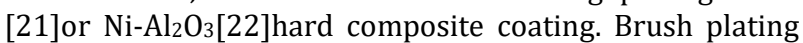
is preferred in the sense ofon-site repair engineering, under which circumstances the main problem for plating is the inferior bonding strength between coating and base, normally on the order of $10 \mathrm{MPa}$. Furthermore, the processing efficiency is low due to the ion deposition, resulting in coating layer thickness usually below $100 \mu \mathrm{m}$. Overlay deposition by thermal spraying or laser cladding provides more effective and practical solutions, particularly for in-filed repair situation of hydraulic components. Hard facing by plasma or laser cladding or welding has been widely investigated in cavitation behaviors. Singh et al [19] studied the cavitation behavior of stellite 6 by laser cladding. The cavitation resistance was enhanced 90\% compared with AISI 304 stainless steel. Santa et al [20] investigated the cavitation resistance of welding martensitic and austenitic steel coating respectively, in which the better cavitation resistance of the later was attributed to high plastic deformation in lab testing. Moreover, it is highly efficient to acquire coatings with thickness on the millimeter scale by cladding or welding. However, cracking tendency of martensite base is considerably high because of the large amount of energy input during cladding or welding.

Thermal spray is a moderately versatile technique for obtaining reasonable property balance of high bonding strength, thickness, cavitation resistance and on-site repair feasibility. Nascimento et al [15] comparatively studied the wear behavior of WC coating deposited by means of high velocity oxygen fuel spraying (HVOF) with chromium plating. The results showed that the former presented better performance in weight loss and fatigue tests. Besides, investigations have also been carried out on the effects of the processing parameters on the wear behaviors and corresponding mechanisms of WC coating by HVOF [23-26].Recently, extensive interest has been focused on HVOF WC coating in anti-cavitation application of hydraulic components. Kumar et al [14] summarized 21 coatings used for repairing cavitation damage in turbines and bumps. They found out that the Stellite 6 by HVOF showed superior cavitation resistance. Taillon et al [18] compared the cavitation behaviors of WC-CoCr and $\mathrm{Cr}_{3} \mathrm{C}_{2}-\mathrm{NiCr}$ cermet deposited by HVOF. Mann et al [16] showed that HVOF WC coating was an excellent erosion shield in hydraulic application in case the coating was highly dense.

By simulation testing of the cavitation impact effect in practical case, the ultrasonic vibratory cavitation erosion is 
an accelerated lab testing technique for cavitation resistance and is standardized in ASTMG32 [27-28]. In this study we present the characterization of the highly dense WC-CoCr coating prepared by HVOF. The cavitation behavior is studied with a direct ultrasonic apparatus according to ASTM G32. The cavitation resistance of WC coating is related with the microstructure and the erosion mechanism is traced by the observation of the worn surface morphology. The results indicate the WC coating by HVOF has a promising anti-cavitation application for hydraulic components.

\section{Experimental}

\section{Materials and coating process}

AISI 410 martensitic stainless steel (410 SS), a typical material for hydraulic turbine blade because of the optimized combination of hardness and toughness, was used as the substrate material for coating and for comparative study of the cavitation performance. The commercial WC-CoCr powder (HC Stack) was chosen as the feedstock for later HVOF spraying. The powder was prepared by water atomization, with diameter distribution being in the range of $10-45 \mu \mathrm{m}$. The nominal chemical composition was $10 \mathrm{wt} \% \mathrm{Co}, 4 \mathrm{wt} \% \mathrm{Cr}$ and the balance WC. The powder was deposited on 410 SS plate with dimensions of $40 \mathrm{~mm} \times 40 \mathrm{~mm} \times 15 \mathrm{~mm}$ by HVOF spraying using the Praxair JP8000 system. The typical spraying parameters were as follows: oxygen flow of $0.94 \mathrm{~m}^{3} / \mathrm{min}$, kerosene flow rate of $0.45 \mathrm{~L} / \mathrm{min}$, gun moving speed of 300 $\mathrm{mm} / \mathrm{s}$. Prior to the spraying, the substrates were cleaned with methanol and sandblasted with alumina (grit 60).In the case of on-site repair spraying for hydraulic components, post-treatments such as heat treatment or mechanical machining or polishing are hardly performed. In accordance with the real hydraulic application, no postspraying treatment was carried on deposited surfaces for later cavitation erosion experiment in this study.

\section{Microstructure characterization}

Powder morphology was characterized by using scanning electron microscopy (SEM, Tescan VEGA TS 5136XM). The powder was observed from both surface and crosssectional view. In the latter case, the large amount of powder particles were embedded in epoxy resin, ground with 1200\# SiC paper and mechanically polished with $1 \mu \mathrm{m}$ diamond suspension to reveal the internal microstructure of the powder.

Coating porosity was evaluated using the software ImageJ from the cross-sectional morphology by optical microscopy (Zeiss Axiover 200 MAT) according to ASTM E2109-00 standard. The specimen was prepared by electrical discharge cutting, mounting, grinding with sandpaper $1200 \#$ and mechanical polishing in sequence following the standard ASTM E1920-03. The phases of the feedstock powder and as-deposited coating were comparatively analyzed by the $\theta / 2 \theta$ diffraction profiles obtained from Xray diffraction (PANalytical X'Pert Pro).A $\mathrm{Cr} K_{\alpha}$ radiation source was used, with a wavelength of $0.2291 \mathrm{~nm}$. Phase identification was accomplished by comparing the sample diffractograms with the International Center for Diffraction Database (ICDD).

\section{Microhardness testing}

Hardness is a direct gauge for evaluating cavitation behavior. The coating microhardness was measured by using Vickers tester (Wilson Worlpert 402 MVD) with the load of $2.94 \mathrm{~N}$ for the duration time of $10 \mathrm{~s}$. The testing was carried out in the cross-section of the coating, prepared following the same procedure as for porosity measurement.

\section{Cavitation testing}

The cavitation erosion test was carried out by ultrasonic vibratory system according to ASTM G32. The test set-up was schematically illustrated in Fig. 1a. The testing specimen was mounted directly on the horn, connecting with sonotrode and transducer. The diameter of the specimen for cavitation test was the same as the horn, being $16 \pm 0.1 \mathrm{~mm}$ as shown in the subfigure. The coating thickness was around $300-400 \mu \mathrm{m}$. The vibrating horn oscillates at a frequency of $20 \pm 0.2 \mathrm{kHz}$ for an amplitude of $50 \pm 2.5 \mu \mathrm{m}$. The horn and specimen were immersed in distilled water cooling system at $20 \pm 2^{\circ} \mathrm{C}$ by a closed loop temperature controller.
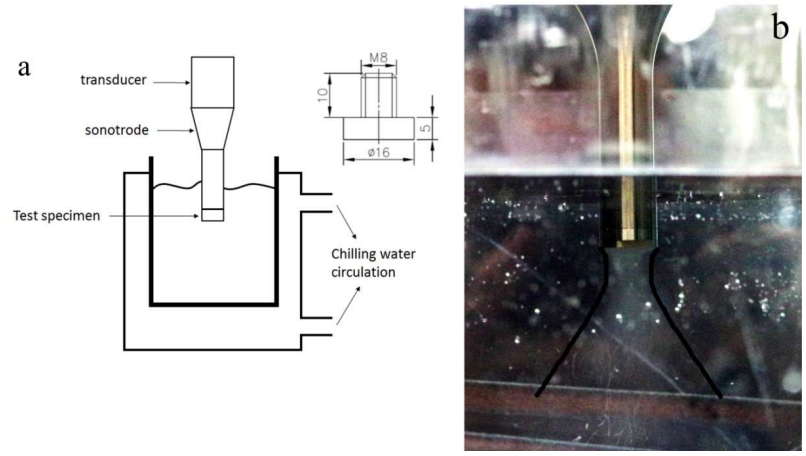

Figure 1: (a) Schematic configuration of the direct ultrasonic vibratory cavitation, the inset graph showing the dimensions of the testing sample; (b) the testing bubble underneath the horn

Large amounts of bubbles were produced by ultrasonic vibrating through the cavitation testing. The bubbles were in the pear-shaped form direct underneath the testing specimen as indicated by the outlines in Fig. 1b. Due to the pressure difference, the bubbles collapsed on the bottom surface of the testing specimens, producing cavitation erosion effect analogous to the working hydraulic components.

The mass change of tested specimen was measured along with cavitation testing time duration of every one hour. The cavitation resistance was quantitatively evaluated by accumulated mass loss, in which three samples were repetitively tested to obtain an average value. The eroded surface morphology was analyzed with white-light interferometry (WLI, Bruker ContourGT) and SEM. The surface roughness was also evaluated by WLI via the arithmetic mean value $R_{\mathrm{a}}$.

\section{Results and Discussion \\ Microstructure}

Figure 2 shows the morphology of the feedstock WC-CoCr powder. From views of both surface (Fig. 2a-b) and crosssection (Fig. 2c-d) directions, the powder particles exhibit good spherical shapes by agglomeration and sintering processing, with only few satellite particles. The diameter distributes homogeneously in the range of 10-450m. From the detailed internal microstructure observation in Fig. 2c$\mathrm{d}$, few obvious ten-micrometer sized pores (arrow in Fig. 2c) and tiny micrometer pores (arrow in Fig. 2d) are 

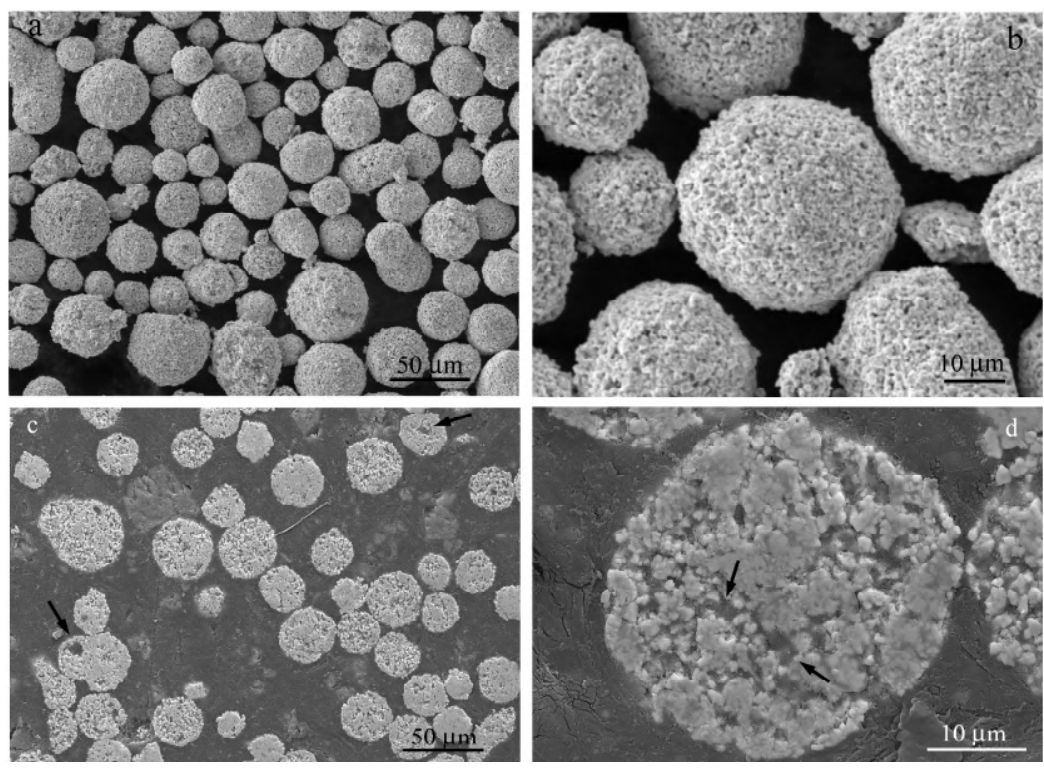

Figure 2: (a-b) surface observation and (c-d) cross-sectional views of the internal microstructure of WC-CoCr feedstock powder
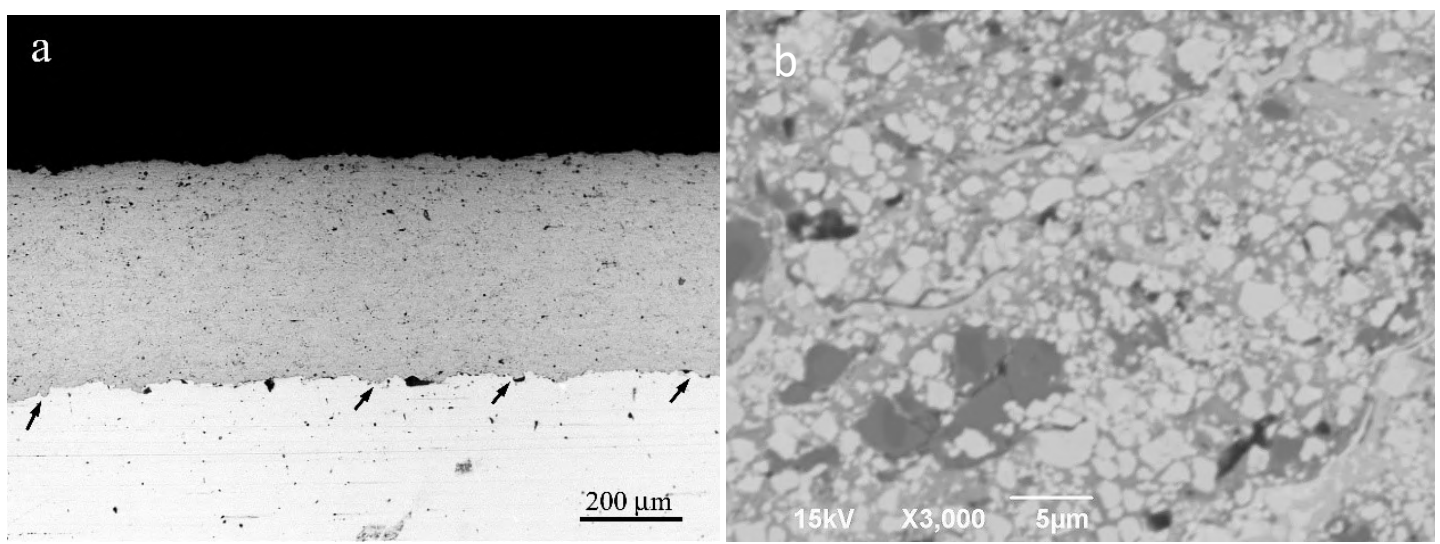

Figure 3: (a) Overview and (b) close observations of the as-sprayed WC-CoCr coating in cross-section

indicated. In close observation (Fig. 2d) the WC phase (dark area) are well embedded in binding CoCr phase (light area).

Figure 3 shows the cross-sectional overview (Fig. 3a) and magnified observation (Fig. 3b) of the as-deposited WCCoCr coating by HVOF processing. The upper coating area shows a uniform thickness, being around $400 \mu \mathrm{m}$. The coating is well bonded with the substrate by the mechanical interlocking as indicated by the arrows (Fig. $3 a$ ). The coating is highly dense with porosity around $0.8 \%$ by imaging analysis. Further observation by SEM (Fig. 3b) reveals no evident boundary among sprayed splats, indicating that the inside splats are well inherently bonded. Furthermore, Fig. $3 \mathrm{~b}$ shows a multi-phases mixed morphology in the coating, where the areas of light grey being WC hard phase and dark areas being CoCr bonding phase.

The wear resistance of $\mathrm{WC}-\mathrm{CoCr}$ coating is highly dependent upon the hard WC phase. However, due to the heat input during spraying, the WC phase in primary powder will be probably decomposed into $\mathrm{W}_{2} \mathrm{C}$ or $\mathrm{W}$ phases, which are detrimental to the toughness of the coating and thus harmful to the cavitation resistance. Fig. 4 compares the X-ray diffraction profiles of the WC-CoCr feedstock powder and the as-deposited coating. It shows that there exists only WC and CoCr phase in the primary feedstock powder. In comparison, the coating phases consist of $\mathrm{WC}, \mathrm{CoCr}$ and $\mathrm{Co}_{3} \mathrm{~W}_{3} \mathrm{C} . \mathrm{Co}_{3} \mathrm{~W}_{3} \mathrm{C}$ is a type of intermediate phase, which was probably formed due to $\mathrm{WC}$ decarburization and then reacting with Co. The broadening of the peaks indicates that the phase is highly in amorphous or nanosized state. No oxides or $\mathrm{W}_{2} \mathrm{C}$ are detected. The X-ray profiles indicate that the coating is well retained as the composite structure of the hard ceramic embedded in tough metallic matrix.

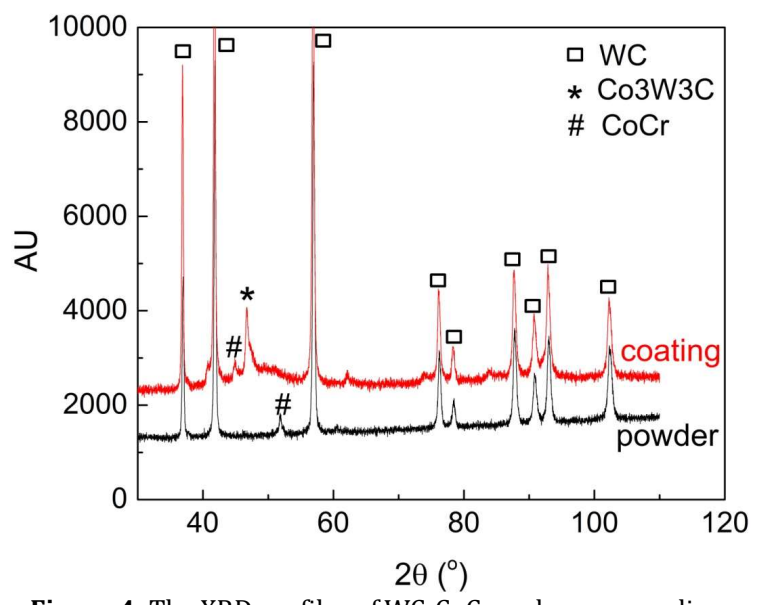

Figure 4: The XRD profiles of WC-CoCr and corresponding HVOF coating 


\section{Cavitation resistance}

The cavitation resistance is directly associated with the hardness. The average hardness of the as-sprayed coating is $12.9 \pm 0.2 \mathrm{GPa}$, which is higher that the documented value of WC-CoCr coating by HVOF in the range of 9-11 GPa [15,29-30]. Furthermore, no cracks are observed at the edges of the indent corners, indicating a favorable toughness of the coating. The $20-60 \%$ higher hardness value combined with good toughness in the coating can be attributed to the ultra-low porosity, the well bonding of hard WC phase within CoCr phase in the coating, and no brittle $\mathrm{W}_{2} \mathrm{C}$ phase. High hardness without cracking indicates that the coating is capable of absorbing large amounts of impact energy during cavitation erosion.

The cavitation resistance is represented by the curves of the accumulative weight loss against testing time, as shown in Fig. 5. It can be largely divided into two stages in both samples, the slow rate of incubation stage, and accelerating stage of high rate. The latter stage can be reflected by a linear relationship between weight losses against time. The incubation time is evaluated at the intercept of the abrupt slope change. The incubation period is about $4 \mathrm{~h}$ and $7 \mathrm{~h}$ for $410 \mathrm{SS}$ and WC-CoCr coatings, respectively, as shown in the inset figure. After the incubation the cavitation rate highly increases. At the testing end, the WC-CoCr weight loss is only half that of $410 \mathrm{SS}$. Testing by the same testing method (ASTM G32), Tailon et al [18] have examined the cavitation behaviors of two typical cermet coatings of $\mathrm{Cr}_{3} \mathrm{C}_{2}-\mathrm{NiCr}$ and WC-CoCr by HVOF. The weight loss of WC-CoCr coating is about $25 \mathrm{mg}$ after testing for $8 \mathrm{~h}$. In comparison, the weight loss in this work is about $18 \mathrm{mg}$, only about $70 \%$ of the same counterpart coating.

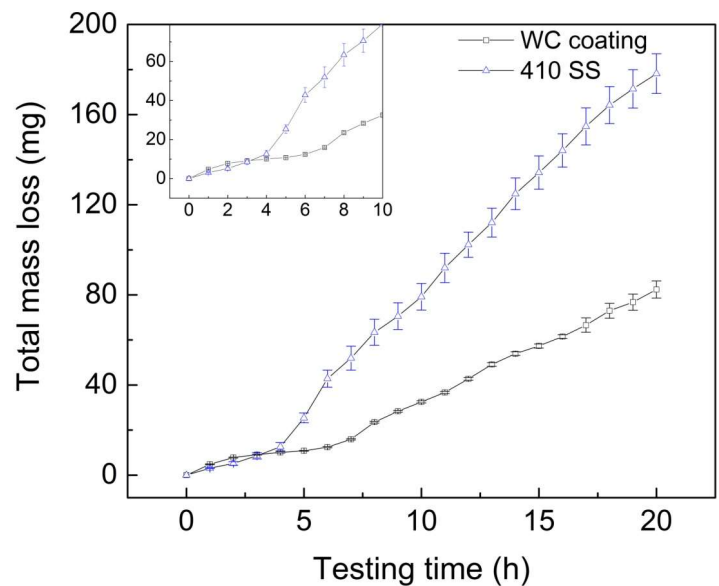

Figure 5: The weight loss against testing time of the WC coating and 410 SS in the cavitation test

\section{Morphology of the worn surfaces}

Figure 6 shows the surface morphology as well as the cross-sectional profiles before and after cavitation testing for $20 \mathrm{~h}$. Since no polish after spraying, the surface roughness $R_{a}$ value of the as-sprayed coating is around 5 $\mu \mathrm{m}$. For comparison the $R_{a}$ of as-polished 410 SS is only $0.09 \mu \mathrm{m}$. After ultrasonic cavitation for $20 \mathrm{~h}$, obvious depressions occur on the surface as shown by the blue spots in Fig. 6b. The surface roughness $R_{a}$ of $410 \mathrm{SS}$ thus increases to $6 \mu \mathrm{m}$. In comparison, the $\mathrm{R}_{\mathrm{a}}$ value of the tested coating increases to $11.9 \mu \mathrm{m}$. Fig. 5-6 shows evidently the depth and diameter of the depressions of 410 SS and WC coating, respectively. The depression depth in 410 SS distributes in the range of $20-50 \mu \mathrm{m}$, with diameter of several hundred microns to over $1 \mathrm{~mm}$. The depression in the WC coating is comparatively larger, with depth around $70 \mu \mathrm{m}$ and diameter over $1 \mathrm{~mm}$.

The worn surface is further observed with SEM, as shown in Fig. 7a-c for 410 SS and Fig. 7d-f for WC coating. After cavitation testing, the surface of both samples has eroded into two morphologies, deep craters and shallow wrinkles. Craters are dispersed rather evenly in the wrinkles (Fig. 7a and $7 d$ ). The lateral size of the craters is in the range of tens of microns, as highlighted in Fig. $7 \mathrm{~b}$ and $7 \mathrm{e}$. The size is much smaller compared to the depressions reflected by WLI (Fig. 6b and 6d).Wrinkles are characterized with chipped shape in the forms of microcracks or tiny embryo craters as indicated by the arrows in Fig. 7c and $7 \mathrm{f}$ respectively. The wrinkles are probably the depressions observed by WLI (Fig. 6b and 6d) for the similar size scale. From the above worn surface examination after the ultrasonic cavitation erosion testing, it can be imaged that a multi-scale pitting-type erosion process has occurred for both $410 \mathrm{SS}$ and WC-CoCr HVOF coating. Shallow wrinkles will foremost form under ultrasonic impacting during the incubation period. These microcracks (Fig. 7c) and embryos (Fig. 7f) form during this period and function as the nucleation zones for later deep crater formation. Then in the second accelerating stage deep craters form through bumps blast, which produces high amounts of weight loss. For 410SS the plastic deformation of martensite microstructure contributes to energy absorption and then cracking results in weight loss. In WC-CoCr coating, the hard ceramic with toughness metallic base poses other mechanisms for cavitation resistance. The plastic deformation of $\mathrm{CoCr}$ binder phase contributes mostly during incubation period as in the case of 410SS. However, in the second stage, there is a selective removal WC phase from CoCr binder because of its high hardness. This starts from the formation of tiny embryo craters (Fig. 7f). The preferential removal of WC induces the high stress concentration at the embryo sites, which makes the enlargement of the craters and explains the weight loss in the second stage.

\section{Conclusions}

A dense WC-CoCr cermet coating was obtained by HVOF spraying. The WC-CoCr coating shows only half weight loss that of 410 SS in direct ultrasonic cavitation erosion testing. The low erosion rate of the coating can be attributed to a combination of ultrahigh hardness and good toughness. The erosion of the coating shows two-stage weight loss behavior, as reflected by the surface morphology of wrinkles and craters. The erosion mechanism is the removal of WC phase and plastic deformation of CoCr metal phase. The results in this work can guide the coating application in hydraulic components.

\section{Acknowledgement}

Financial support from National Natural Science Foundation of China (Grant No. 51501120), Natural Science Foundation of Jiangsu Province (Grant No. BK20150335), Natural Science Foundation for Universities in Jiangsu Province (Grant No. 15KJB430029) and Suzhou Science and Technology Program (SGC201720)is acknowledged. 

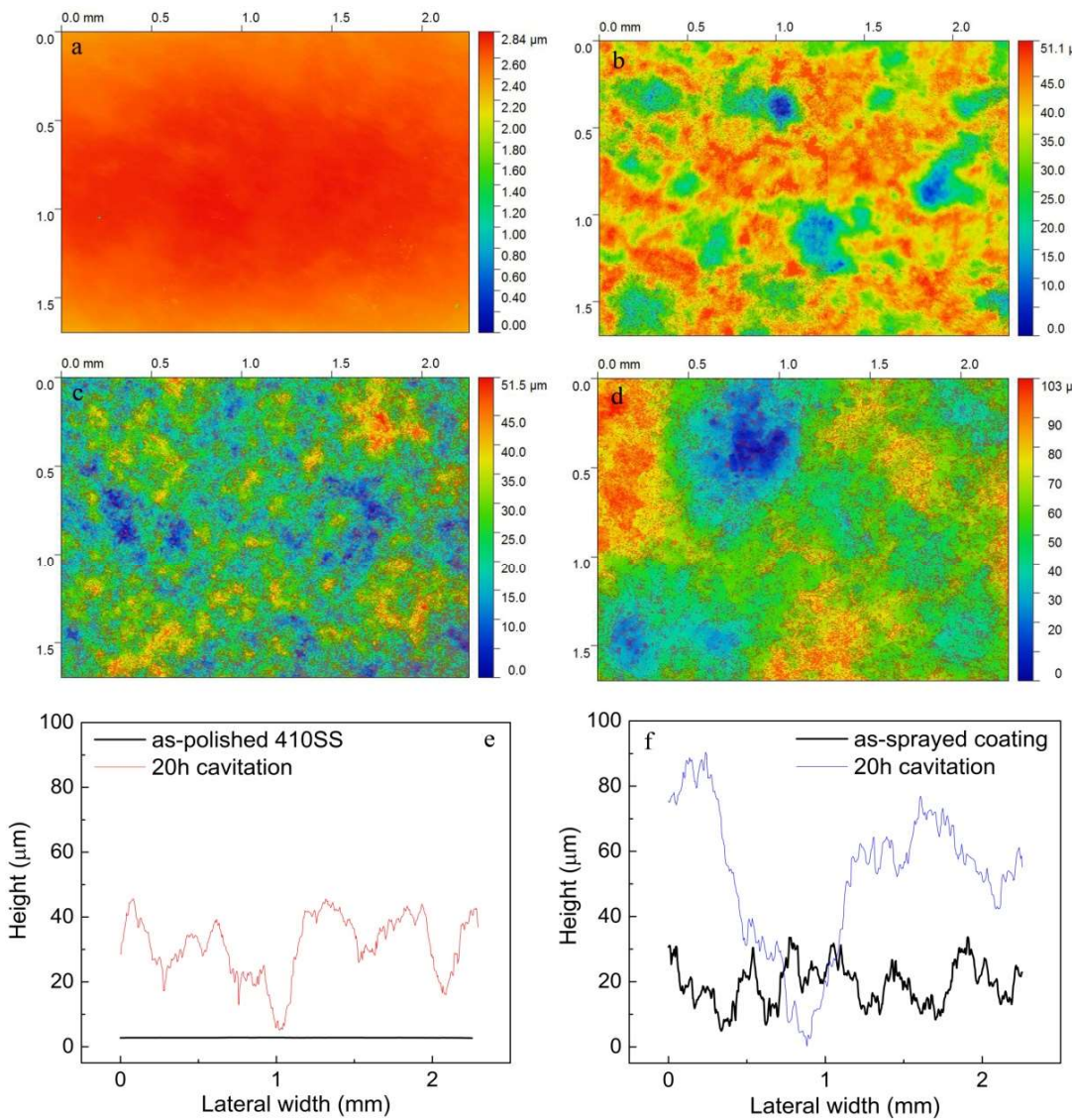

Figure 6: The surface morphology by white light interferometry: (a) as-polished 410 SS; (b) 410 SS after 20 h cavitation testing (c) as-sprayed WC coating; (d) WC coating after $20 \mathrm{~h}$ cavitation testing. And the cross-sectional profiles before and after testing: (e) $410 \mathrm{SS}$, (f) WC coating
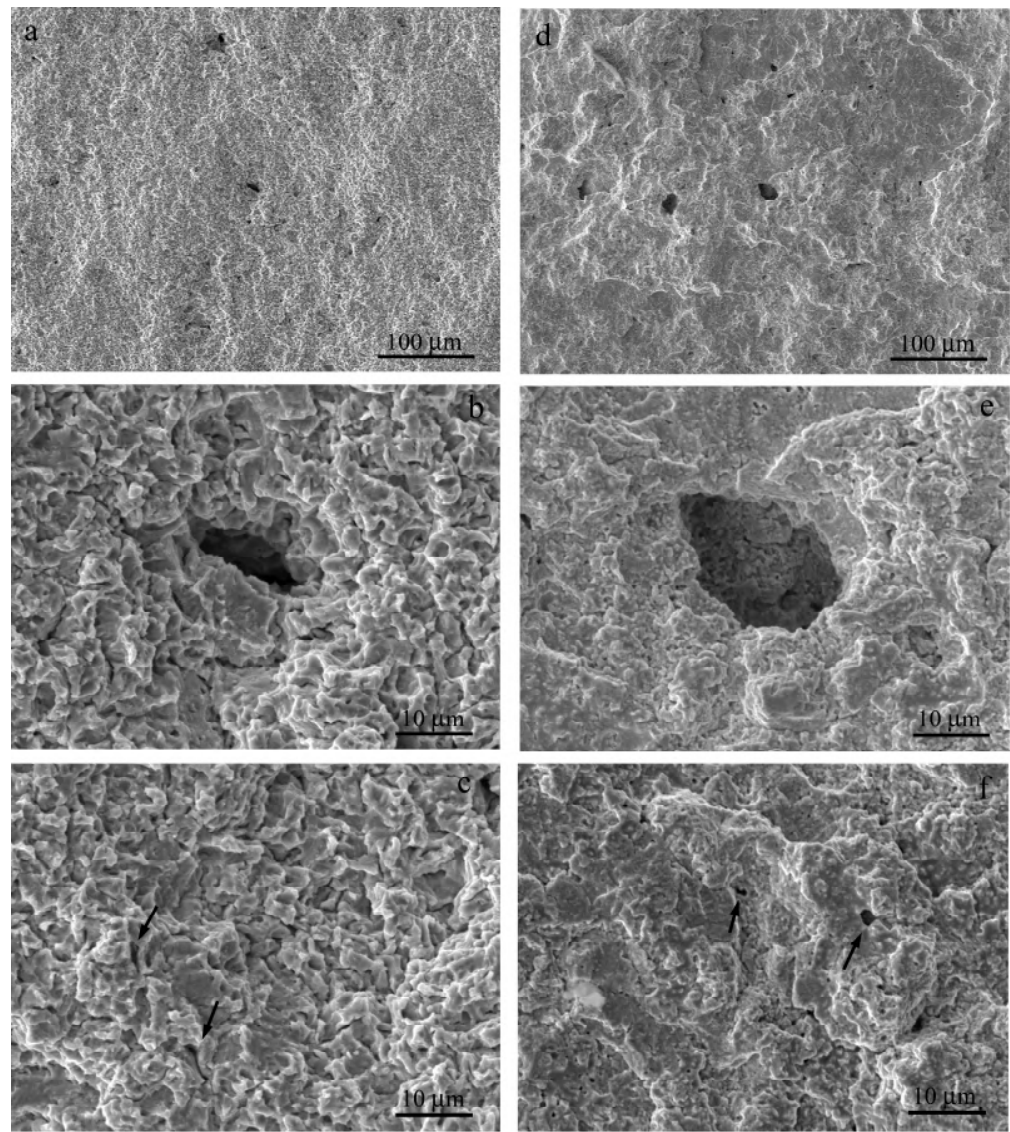

Figure 7: Overview and close observations of the $410 \mathrm{SS}$ (a-c) and WC-CoCr coatings (d-f) after cavitation testing for $20 \mathrm{~h}$ 


\section{References}

1. S.C. Li, Cavitation of Hydraulic Machinery, Imperial College Press, 2001.

2. P. Kumar, R.P. Saini, Study of cavitation in hydro turbines - A review, Renew Sust Energ Rev, 2010, 14(1), 374-83.

3. B.K. Sreedhar, S.K. Albert, A.B. Pandit, Cavitation damage: Theory and measurements - A review, Wear, 2017, 372-373, 177-96.

4. R.T. Knapp, J.W. Daily, F.G. Hammit, Cavitation, McGraw-HIll, 1970.

5. M. ČDina, Detection of cavitation phenomenon in a centrifugal pump using audible sound, Mech Syst Signal Process, 2003, 17(6), 1335-47.

6. O. Coutier-Delgosha, R. Fortes-Patella, J.L. Reboud, M. Hofmann, B. Stoffel, Experimental and numerical studies in a centrifugal pump with two-dimensional curved blades in cavitating condition, J Fluid Eng, 2004, 125(6), 970-78.

7. Y.-c. Peng, X.-y. Chen, Y. Cao, G.-x. Hou, Numerical study of cavitation on the surface of the guide vane in three gorges hydropower unit, J Hydrodynam B, 2010, 22(5), 703-08.

8. M.V. Timoshevskiy, S.A. Churkin, A.Y. Kravtsova, K.S. Pervunin, D.M. Markovich, K. Hanjalić, Cavitating flow around a scaled-down model of guide vanes of a highpressure turbine, Int J Multiph Flow, 2016, 78, 75-87.

9. K. Celebioglu, B. Altintas, S. Aradag, Y. Tascioglu, Numerical research of cavitation on Francis turbine runners, Int J Hydrog Energy, 2017, 42(28), 17771-81.

10. A. Luna-Ramírez, A. Campos-Amezcua, O. Dorantes-Gómez, Z. Mazur-Czerwiec, R. Muñoz-Quezada, Failure analysis of runner blades in a Francis hydraulic turbine - Case study, Eng Fail Anal, 2016, 59, 314-25.

11. C.J. Lin, J.L. He, Cavitation erosion behavior of electroless nickel-plating on AISI 1045 steel, Wear, 2005, 259(1), 15459.

12. Y. Iwai, T. Okada, T. Fujieda, K. Awazu, Effects of hard chromium plating on cavitation erosion, Wear, 1988, 128(2), 189-200.

13. T. Okada, Y. Iwai, K. Awazu, Effects of plating on cavitation erosion, Wear, 1988, 124(1), 21-31.

14. A. Kumar, J. Boy, R. Zatorski, L.D. Stephenson, Thermal spray and weld repair alloys for the repair of cavitation damage in turbines and pumps: A technical note, J Therm Spray Technol, 2005, 14(2), 177-82.

15. M.P. Nascimento, R.C. Souza, I.M. Miguel, W.L. Pigatin, H.J.C. Voorwald, Effects of tungsten carbide thermal spray coating by HP/HVOF and hard chromium electroplating on AISI 4340 high strength steel, Surf Coat Technol, 2001, 138(2-3), 113-24.

16. B.S. Mann, V. Arya, Abrasive and erosive wear characteristics of plasma nitriding and HVOF coatings: their application in hydro turbines, Wear, 2001, 249(5), 354-60.

17. R.K. Kumar, M. Kamaraj, S. Seetharamu, T. Pramod, P. Sampathkumaran, Effect of spray particle velocity on cavitation erosion resistance characteristics of hvof and hvaf processed 86wc-10co4cr hydro turbine coatings, J Therm Spray Technol, 2016, 25(6), 1217-30.

18. G. Taillon, F. Pougoum, S. Lavigne, L. Ton-That, R. Schulz, E. Bousser, S. Savoie, L. Martinu, J.-E. Klemberg-Sapieha, Cavitation erosion mechanisms in stainless steels and in composite metal-ceramic HVOF coatings, Wear, 2016, 364365, 201-10.

19. R. Singh, D. Kumar, S.K. Mishra, S.K. Tiwari, Laser cladding of Stellite 6 on stainless steel to enhance solid particle erosion and cavitation resistance, Surf Coat Technol, 2014, 251, 8797.

20. J.F. Santa, J.A. Blanco, J.E. Giraldo, A. Toro, Cavitation erosion of martensitic and austenitic stainless steel welded coatings, Wear, 2011, 271(9), 1445-53.

21. Z. Zhong, S.J. Clouser, Nickel-tungsten alloy brush plating for engineering applications, Surf Coat Technol, 2014, 240, 38086.

22. B. Wu, B.-s. Xu, B. Zhang, X.-d. Jing, C.-l. Liu, Automatic brush plating: An update on brush plating, Mater Lett, 2006, $60(13), 1673-77$.
23. J.A. Picas, M. Punset, M.T. Baile, E. Martín, A. Forn, Effect of oxygen/fuel ratio on the in-flight particle parameters and properties of HVOF WC-CoCr coatings, Surf Coat Technol, 2011, 205, S364-S68.

24. T. Sahraoui, S. Guessasma, M. Ali Jeridane, M. Hadji, HVOF sprayed WC-Co coatings: Microstructure, mechanical properties and friction moment prediction, Mater Des, 2010, 31(3), 1431-37.

25. C. Thiruvikraman, V. Balasubramanian, K. Sridhar, Optimizing HVOF Spray Parameters to Maximize Bonding Strength of WC-CrC-Ni Coatings on AISI 304L Stainless Steel, J Therm Spray Technol, 2014, 23(5), 860-75.

26. Q. Wang, Z. Chen, L. Li, G. Yang, The parameters optimization and abrasion wear mechanism of liquid fuel HVOF sprayed bimodal WC-12Co coating, Surf Coat Technol, 2012, 206(8), 2233-41.

27. ASTM, "Standard Test Method for Cavitation Erosion Using Vibratory Apparatus," ASTM International 2016.

28. K.-H. Kim, G. Chahine, J.-P. Franc, A. Karimi, Advanced Experimental and Numerical Techniques for Cavitation Erosion Prediction, Springer Netherlands, 2014, p 21-35.

29. J.K.N. Murthy, B. Venkataraman, Abrasive wear behaviour of WC-CoCr and $\mathrm{Cr} 3 \mathrm{C} 2-20(\mathrm{NiCr})$ deposited by HVOF and detonation spray processes, Surf Coat Technol, 2006, 200(8), 2642-52.

30. S. Al-Mutairi, M.S.J. Hashmi, B.S. Yilbas, J. Stokes, Microstructural characterization of HVOF/plasma thermal spray of micro/nano WC-12\%Co powders, Surf Coat Technol, 2015, 264, 175-86. 